\title{
Standard-Dose Rituximab as Effective Therapy for Treating Malignancy-Related Hemophagocytic Lymphohistiocytosis in the Eldery: A Case Report
}

\author{
Blessie Elizabeth Nelson ${ }^{a, b} \quad$ Angelina Hong $^{b} \quad$ Mhair Dekmezian $^{c}$ \\ Bagi Janad \\ aDepartment of Hematology \& Oncology, University of Texas Medical Branch, Galveston, \\ TX, USA; bSchool of Medicine, University of Texas Medical Branch, Galveston, TX, USA; \\ 'Department of Pathology, Mainland Medical Center, Texas City, TX, USA; dDepartment of \\ Hematology \& Oncology, MD Anderson Cancer Center, Houston, TX, USA
}

\section{Keywords}

Hemophagocytic lymphohistiocytosis · Chronic lymphocytic leukemia · Geriatrics · Elderly

\section{Abstract}

Hemophagocytic lymphohistiocytosis $(\mathrm{HLH})$ is a syndrome involving uncontrolled inflammation due to widespread activation of immune response. HLH can be inherited or acquired secondary to infection, autoimmune, or oncologic processes such as small lymphocytic lymphoma (SLL) or chronic lymphocytic leukemia (CLL). There has been minimal documentation of HLH secondary to SLL/CLL, and results of treatment have been largely unsuccessful. This case describes a critically ill elderly patient with HLH caused by SLL/CLL who was successfully treated with standard-dose rituximab and regained a high quality of life.

(c) 2021 The Author(s).

Published by S. Karger AG, Basel

\section{Case Report}

An 80-year-old female with a history of rheumatoid arthritis, hypertension, aortic stenosis, and hypothyroidism presented with fatigue, shortness of breath, and lower extremity edema. She was hypotensive and required vasopressors in the ICU before being transferred to the floor. She exhibited high-grade fever, pancytopenia, with hemoglobin (HGB) $6.9 \mathrm{~g} / \mathrm{dL}$, 
Nelson et al.: Treating Malignancy-Related Hemophagocytic Lymphohistiocytosis in the Elderly

platelet (PLT) count 33,000/ $\mu \mathrm{L}$, white blood cell count (WBC) $790 / \mu \mathrm{L}$, and absolute neutrophil count $540 / \mu \mathrm{L}$. She was admitted twice in the past 2 months for anemia and required transfusions during this hospitalization. Her recent bone marrow biopsy indicated that she had small lymphocytic lymphoma (SLL). Her Eastern Cooperative Oncology Group (ECOG) performance status was 4 . Subsequent workup showed that the patient had hemophagocytic lymphohistiocytosis (HLH), as she had splenomegaly, cytopenia, bone marrow involvement with hemophagocytosis as depicted in Figure 1, elevated serum interleukin-2 receptor levels, elevated serum triglyceride level of $261 \mathrm{mg} / \mathrm{dL}$, and an elevated serum ferritin level of 4,960 ng/mL; thus, she met 5 of the 8 diagnostic criteria. Induction chemotherapy was started. Because of the patient's poor renal function, bendamustine was held in the first cycle, and rituximab alone was given at $375 \mathrm{mg} / \mathrm{m}^{2}$.

Following initiation of rituximab, patient's pancytopenia improved, with WBC $2760 / \mu \mathrm{L}$, absolute neutrophil count $600 / \mu \mathrm{L}$, and PLT $48,000 / \mu \mathrm{L}$ and ferritin decreased to 2,550 ng/ $\mathrm{mL}$. Her HGB was stable at $9.4 \mathrm{~g} / \mathrm{dL}$, and she did not require further transfusions. She no longer complained of fatigue or shortness of breath. 3 weeks after discharge, the patient continued to improve with WBC $4,110 / \mu \mathrm{L}$ and PLT count $149,000 / \mu \mathrm{L}$ and HGB $9.3 \mathrm{~g} / \mathrm{dL}$. She had no symptoms, and ECOG score returned to 0 . She started maintenance therapy with rituximab and bendamustine and had no adverse side effects other than mild fatigue during the nadir of therapy.

\section{Discussion}

This case report demonstrates how standard-dose rituximab can be safely given to critically ill, geriatric patients with HLH secondary to SLL/chronic lymphocytic leukemia (CLL). HLH is caused by the uncontrolled proliferation of CD8+ T cells, which in turn activate histiocytes and macrophages via the release of interferon-gamma. These cells regulate the phagocytosis of blood cells in multiple organs. The diagnosis of HLH is characterized by meeting 5 of the 8 criteria: fever greater than or equal to $38.5^{\circ} \mathrm{C}$, splenomegaly, cytopenias, hypertriglyceridemia and/or hypofibrinogenemia, hemophagocytosis in bone or other tissues, low to absent natural killer cell activity, ferritin $>500 \mathrm{ng} / \mathrm{mL}$, and elevated serum levels of soluble IL-2 receptor. These signs and symptoms are the result of HLH's mass production of inflammatory cytokines, which include interferon-gamma, interleukin-6, interleukin-18, and tumor necrosis factor-alpha [1]. HLH can be familial/primary or acquired/secondary. Primary HLH is autosomal recessive, with incomplete penetrance, and has various subtypes, such as those involving inherited defects in $\mathrm{T}$ cell or natural killer cell cytotoxic activity/machinery, perforin deficiency, or increased susceptibility to Epstein Barr Virus (EBV) infection [2]. The most common etiology of secondary HLH is infection by herpes viruses such as EBV but can also be caused by rheumatologic, autoimmune, or oncologic processes [3].

Treatment of HLH involves addressing the underlying problem and limiting hyperinflammation, which is a challenging feat in elderly patients with HLH secondary to SLL/ CLL. However, recent literature and the findings from this case report demonstrate that rituximab can be safely administered to elderly patients with HLH and produce successful outcomes. Rituximab is an anti-CD20 monoclonal antibody that causes complement and antibody-dependent cytotoxicity and apoptosis, which can cause complications ranging from nausea and vomiting to more adverse consequences such as cytopenia, severe infection, renal failure, interstitial lung disease, or arrhythmias [4]. There are limited studies focusing particularly upon chemotherapy outcomes in the geriatric population with SLL/CLL. Shah et al. [5] review found that CLL patients over the age of 70 are

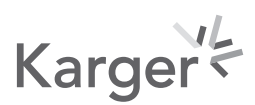




\section{Case Reports in Oncology}

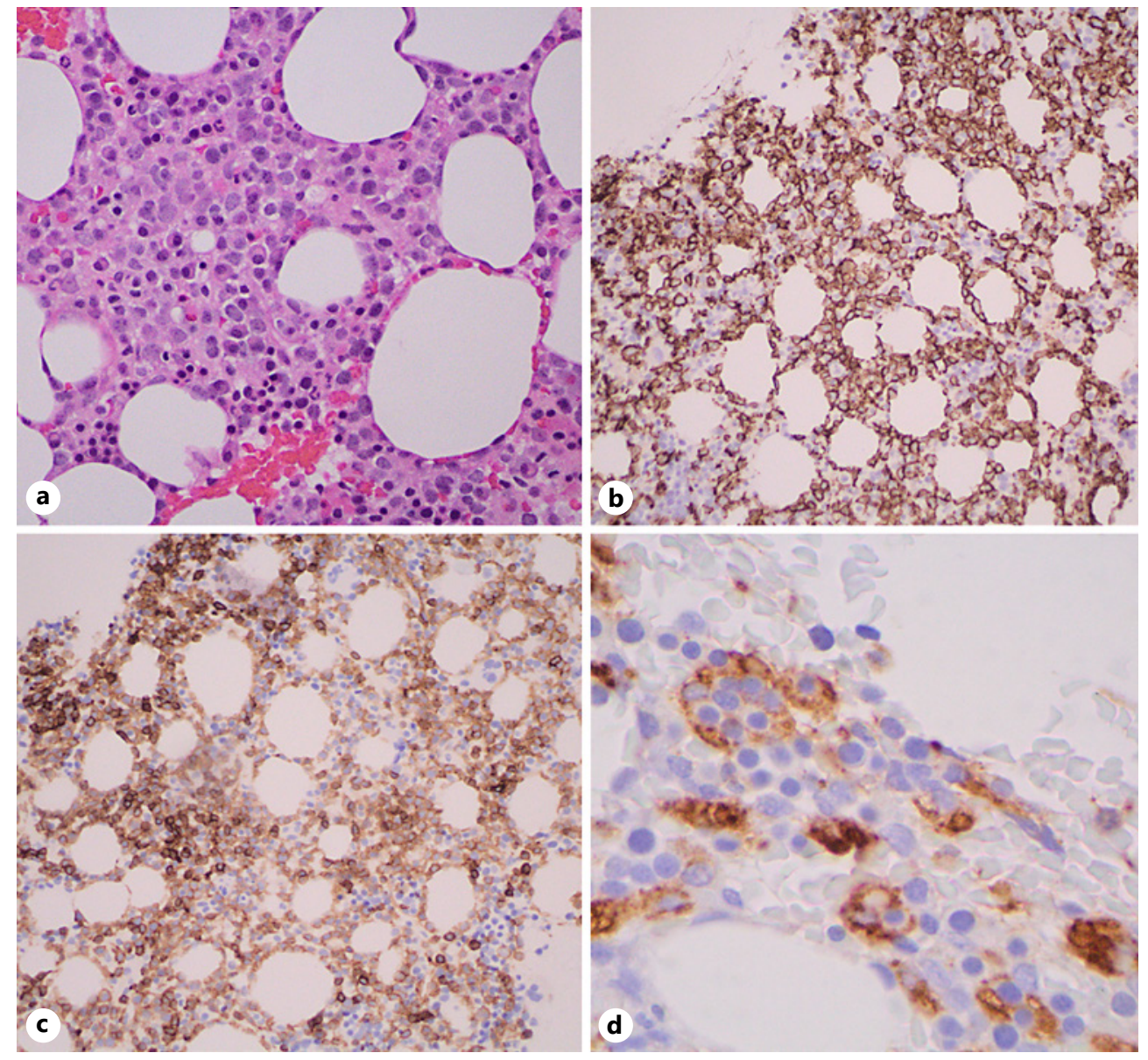

Fig. 1. a The core biopsy shows many small-to-medium mononuclear cells in a background of maturing erythroid and myeloid cells as well as scattered plasma cells (HE. ×400). b CD20 immunohistochemistry shows a diffuse infiltrate within the marrow of B lymphocytes ( $\times 100)$. c CD5 immunohistochemistry shows most of the B lymphocytes expressing weak or moderate expression. Scattered strong positive T lymphocytes are also present $(\times 100)$. Not pictured: cyclinD1 expression is mostly negative, sox11 is negative, and LEF1 is diffusely positive. MYD88 mutation testing is positive for c.794T >C (p.L265P. In situ hybridization shows polytypic kappa and lambda expression by plasma cells. d CD68 immunohistochemistry shows scattered monocytic lineage cells exhibiting phagocytosis of other marrow elements $(\times 1,000)$.

generally more prone to acquiring severe hematologic complications from regimens including rituximab, fludarabine, and/or cyclophosphamide (FCR) but proposes that patients with good organ function and low CIRS and ECOG scores can still be considered as candidates for FCR. In contrast, Herishanu et al. [6] phase II trial demonstrated that FCR involving standard-dose rituximab can be safely used in elderly patients, even in those with relatively high Charlson Age-Comorbidity Index scores. Of the 40 patients treated with FCR, only 3 discontinued treatments due to adverse effects, and one died 8 months after completing treatment. Additionally, Pileckyte et al. [7] found that high-dose rituximab in combination with methylprednisolone can be safely administered to elderly and high-risk CLL patients. Chellapandian et al. [8] study found that rituximab-containing regimens successfully improved symptoms and reduced inflammation in patients with EBV-associated HLH. The median age at diagnosis for these patients was 65-75 years, so most of this study population was geriatric. Thus, the literature suggests that rituximab 
therapy can be safely used for management of HLH in elderly patients with or without comorbidities, malignancy, or an active viral infection. As of now, this case report is the only known documentation of a patient with SLL/CLL and no active infection whose HLH was resolved using one cycle of standard-dose rituximab and experienced no adverse side effects.

There is also some literature suggesting that certain immunotherapy agents such as rituximab used to treat HLH can cause it as well. This is perhaps a reason why there are so few case reports documenting HLH with this etiology because authors are uncertain whether HLH existed before initiating chemotherapy, or if it was the direct result of a malignant progression $[9,10]$. However, the patient described in this case had received no treatment for her CLL/SLL prior to presentation, so her HLH could not have been induced by rituximab or chemotherapy regimens.

\section{Conclusion}

Effective treatment of HLH involves not only short-term stabilization but must also consider the patient's long-term quality of life and potential complications. This case report is an example of how rituximab can be an effective and safe therapy for a geriatric patient with HLH secondary to SLL/CLL. This patient responded strongly to treatment and experienced minimal adverse side effects. This case demonstrates how elderly patients with low-grade lymphoma and HLH can be safely treated with standard-dose rituximab instead of resorting to an aggressive chemotherapy regimen. Our findings support the vital role of rituximab for the treatment of HLH, which emphasizes the need for increased research on this topic in the geriatric population.

\section{Acknowledgements}

The authors would like to thank the Department of Internal Medicine and Critical Care and Infectious Diseases of University of Texas Medical Branch, Galveston, Texas in patient care.

\section{Statement of Ethics}

Written informed consent was obtained from the patient's guardian for publication of this case report and any accompanying images. No Ethical Review Board is required for this publication.

\section{Conflict of Interest Statement}

There are no conflicts of interest.

\section{Funding Sources}

The authors did not receive any funding.

\section{Karger'k}




\section{Case Reports in Oncology}

\begin{tabular}{l|l}
\hline Case Rep Oncol 2021;14:1066-1070 \\
\hline DOI: 10.1159/000517023 & $\begin{array}{l}\text { ○ 2021 The Author(s). Published by S. Karger AG, Basel } \\
\text { www.karger.com/cro }\end{array}$ \\
\hline
\end{tabular}

Nelson et al.: Treating Malignancy-Related Hemophagocytic Lymphohistiocytosis in the Elderly

\section{Author Contributions}

B.N. and A.H.: developed the original idea, analyzed the data, prepared the manuscript, and provided additional review. M.D.: contributed to the development of the manuscript and prepared the manuscript. B.J.: provided critical revision of the manuscript for important intellectual content.

\section{References}

1 Risma KA, Marsh RA. Hemophagocytic lymphohistiocytosis: clinical presentations and diagnosis. J Allergy Clin Immunol Pract. 2018;7(3):824-32.

2 de Saint Basile G, Ménasché G, Latour S. Inherited defects causing hemophagocytic lymphohistiocytic syndrome. Ann N Y Acad Sci. 2011;1246:64-76.

3 Esteban YM, de Jong JLO, Tesher MS. An overview of hemophagocytic lymphohistiocytosis. Pediatr Ann. 2017; 46(8):e309-13.

4 Kasi PM, Tawbi HA, Oddis CV, Kulkarni HS. Clinical review: serious adverse effects associated with the use of rituximab: a critical care perspective. Crit Care. 2012;16(4):231.

5 Shah N, Tam C, Seymour JF, Rule S. How applicable is fludarabine, cyclophosphamide and rituximab to the elderly? Leuk Lymphoma. 2015;56(6):1599-610.

6 Herishanu Y, Tadmor T, Braester A, Bairey O, Aviv A, Rahimi-Levene N, et al. Low-dose fludarabine and cyclophosphamide combined with standard dose rituximab (LD-FCR) is an effective and safe regimen for elderly untreated patients with chronic lymphocytic leukemia: the Israeli CLL study group experience. Hematol Oncol. 2019;37:185-92.

7 Pileckyte R, Valceckiene V, Stoskus M, Matuzeviciene R, Sejoniene J, Zvirblis T, et al. Dose intensive rituximab and high-dose methylprednisolone in elderly or unfit patients with relapsed chronic lymphocytic leukemia. Medicina. 2019;55(11):719.

8 Chellapandian D, Das R, Zelley K, Wiener SJ, Zhao H, Teachey DT, et al. Treatment of Epstein Barr virus-induced haemophagocytic lymphohistiocytosis with rituximab-containing chemo-immunotherapeutic regimens. Br J Haematol. 2013;162:376-82.

9 Meki A, O'Connor D, Roberts C, Murray J. Hemophagocytic lymphohistiocytosis in chronic lymphocytic leukemia. J Clin Oncol. 2011;29(24):e685-7.

10 Tiong IS, Lau MB, Toumoua S, Chiruka S. A case of hemophagocytic lymphohistiocytosis in a patient with chronic lymphocytic leukemia after treatment with fludarabine, cyclophosphamide, and rituximab chemotherapy, with autopsy findings. Case Rep Hematol. 2012;2012:326053. 